\title{
Imaging Algorithm of Missile-borne SAR in Diving and Squint Mode
}

\author{
Zhen Wang, Xiaodong He and Bin Tang \\ School of Electronic Engineering, University of Electronic Science and Technology of China, Sichuan, China \\ jeannewang1990@gmail.com
}

\begin{abstract}
In this paper, we propose an imaging algorithm of missile-borne SAR in diving and squint mode. The diving acceleration, vertical speed, and squint angle caused a large range cell migration (RCM). The algorithm divides the RCM correction (RCMC) into two steps. Firstly, correcting the main part of range walk in time domain. Secondly, correcting the remaining RCMC in range frequency-azimuth Doppler frequency domain, using an analytical form of the signal 2-D spectrum derived by the method of series reversion. Theoretical analysis and simulation results illustrate its validity, and satisfy the imaging quality of missile-borne SAR in high squint model.

Index Terms - Missile-borne SAR, diving and squint mode, RCMC, series reversion.
\end{abstract}

\section{Introduction}

Compared with general airborne or space-borne SAR, missile-borne SAR has significantly different characteristics. In order to attack the target, missile-borne SAR cannot do the uniform line motion, and also it has a certain acceleration and vertical velocity in the diving flight. Moreover, it usually works in a squint mode in order to acquire a period of turning time. Therefore, the general SAR imaging algorithms are no longer suitable for missile-borne SAR [1-2].

In [3], the 2-D spectrum of target echo was derived by the method of series reversion, and the RCMC, range and azimuth compression were accomplished in the 2-D frequency domain. But the algorithm was only suitable for the center single point target imaging of the scene in side-looking mode. An imaging algorithm for missile-borne SAR based on azimuth nonlinear chirp scaling (NLCS) was proposed in [4]. After finished RCMC and range compression in the 2-D frequency domain by the method of series reversion, azimuth variation of Doppler FM rates for echo signal were compensated with the operation of azimuth NLCS, which could improve focusing depth and effect. However, the computation is too heavy, and it also only worked in side-looking mode. In [5], the spectral overlapping phenomenon in distance was solved by introducing a new CS factor, and the influence caused by velocity and acceleration of the missile were compensated. A deramp method was introduced to cope with the Doppler spectral overlapping phenomenon in azimuth, but this algorithm was too complex to realize.

According to the characteristics of the missile-borne SAR platform motion, a space geometry relationship of the diving and squint movement is established in this paper. Then the instantaneous range between radar and target is calculated, and the point target echo signal model is derived. The Doppler frequency center is corrected in range frequency-azimuth time domain [6]. The 2-D spectrum of the point target echo signal is deduced using the method of series reversion. After finishing the pulse compression processing, a focused point target image is got. At last, a missile-borne SAR simulation is used to illustrate its validity of the algorithm.

\section{Geometric Model and Instantaneous Range}

The imaging geometry of missile-borne SAR is shown in Fig. 1. A missile is assumed to be flying along the trajectory of $A B C$ with a constant acceleration in a two-dimensional plane, which is locally vertical to the surface of the Earth and the projection of trajectory on the ground is $X$ axis. The range time is given by $t_{r}$, and the azimuth time $t_{a}$ is chosen to be zero at the composite beam center crossing time (mid exposure time) of the reference target. $V$ is the velocity of the missile, and the horizontal and vertical velocity are $V_{x}$ and $V_{z}$, accompanying with the constant acceleration $a_{x}$ and $a_{z}$. While the velocity and acceleration are all zero. $H$ is the height of the missile at $t_{a}=0$. The instantaneous range from SAR to an arbitrary reference point target $P\left(x_{0}, y_{0}, 0\right)$ is $R_{0}$, and The shortest range $R_{c}$ has a relationship with $R_{0}$ and squint angle $\theta$, which is $R_{c}=R_{0} \cos \theta$.

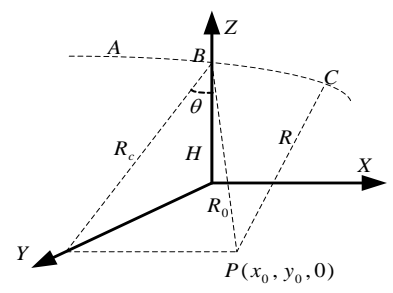

Fig. 1 The diving and squint missile-borne SAR geometry.

The instantaneous range from SAR to a point target at azimuth time $t_{a}$ can be expressed as

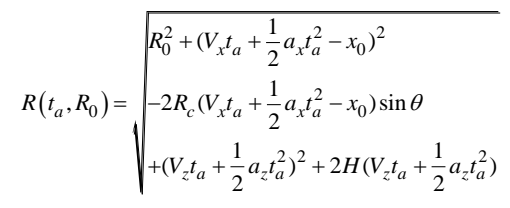

The first 5th order Taylor expansion [7] of (1) can be expressed as 


$$
R\left(t_{a} ; R_{0}\right) \approx k_{00}+k_{10} t_{a}+k_{20} t_{a}^{2}+k_{30} t_{a}^{3}+k_{40} t_{a}^{4}+0\left(t_{a}^{5}\right)
$$

Letting $w_{1}=-V_{x} x_{0}+H V_{z}-R_{c} V_{x} \sin \theta, w_{2}=V_{x}^{2}+V_{z}^{2}-a_{x} x_{0}+H a_{z}-R_{c} a_{x} \sin \theta$, $w_{3}=V_{x} a_{x}+V_{z} a_{z}, w_{4}=a_{x}^{2}+a_{z}^{2}, R_{x}=\sqrt{R_{0}^{2}+x_{0}^{2}+2 R_{c} x_{0} \sin \theta}, a_{1}=-V_{x} x_{0} / R_{x}$. Then, the coefficients in (2) can be expressed as

$$
\left\{\begin{array}{l}
k_{00}=R_{x}, k_{10}=\frac{w_{1}}{R_{x}}=a_{1}+\frac{H V_{z}}{R_{x}}+\frac{-R_{c} V_{x} \sin \theta}{R_{x}}, \\
k_{20}=\frac{w_{2}}{2 R_{x}}-\frac{w_{1}^{2}}{2 R_{x}^{3}}, k_{30}=\frac{w_{3}}{2 R_{x}}-\frac{w_{1} w_{2}}{2 R_{x}^{3}}+\frac{w_{1}^{3}}{2 R_{x}^{5}} \\
k_{40}=\frac{w_{4}}{8 R_{x}}-\frac{w_{2}^{2}+4 w_{1} w_{3}}{8 R_{x}^{3}}+\frac{3 w_{1}^{2} w_{2}}{4 R_{x}^{5}}-\frac{5 w_{1}^{4}}{8 R_{x}^{7}}
\end{array}\right.
$$

\section{Analysis of Signal and Imaging Algorithm}

A. Derivation of the Signal Spectrum Assuming the transmitted LFM signal is

$$
s_{t}\left(t_{r}, t_{a} ; R_{0}\right)=a_{r}\left(t_{r}\right) \exp \left(j 2 \pi f_{c} t_{r}+j \pi \gamma t_{r}^{2}\right)
$$

where $f_{c}$ is the carrier frequency, $\gamma$ is the range chirp rate, $a_{r}(\cdot)$ and is $a_{a}(\cdot)$ are the range and azimuth envelopes.

The echo received from a reference point target experiences a delay that is proportional to the two-way slant range $2 R\left(t_{a} ; R_{0}\right)$. After down-converted to baseband, it can be expressed with range time $t_{r}$ and azimuth time $t_{a}$ as

$$
\begin{aligned}
& s\left(t_{r}, t_{a} ; R_{0}\right)=s_{t}\left(t_{r}-2 R\left(t_{a} ; R_{0}\right) / c, t_{a} ; R_{0}\right) \\
& =a_{r}\left(t_{r}-2 R\left(t_{a} ; R_{0}\right) / c\right) a_{a}\left(t_{a}\right) \\
& \exp \left[-j \frac{4 \pi}{\lambda} R\left(t_{a} ; R_{0}\right)\right] \exp \left[j \pi \gamma\left(t_{r}-2 R\left(t_{a} ; R_{0}\right) / c\right)^{2}\right]
\end{aligned}
$$

where $\lambda$ is the radar wavelength, $c$ is the speed of light.

Using Principal of Stationary Phase (POSP) and Fresnel Integral [8], the range FT of (5) can be expressed as

$$
\begin{aligned}
S\left(f_{r}, t_{a} ; R_{0}\right) & =\int s\left(t_{r}, t_{a} ; R_{0}\right) \exp \left(-j 2 \pi f_{r} t_{r}\right) d t_{r} \\
& =a_{r}\left(f_{r}\right) a_{a}\left(t_{a}\right) \exp \left(-j \frac{\pi f_{r}^{2}}{\gamma}\right) \\
& \exp \left[-j 4 \pi \frac{f_{r}+f_{c}}{c} R\left(t_{a} ; R_{0}\right)\right]
\end{aligned}
$$

where $f_{r}$ is the range frequency. Then the Doppler center frequency of range can be given by

$$
f_{d c}=-\left.\frac{2}{\lambda} \frac{d R\left(t_{a} ; R_{0}\right)}{d t_{a}}\right|_{t_{a}=0}=-\frac{2}{\lambda}\left(a_{1}+\frac{H V_{z}}{R_{x}}-\frac{R_{c} V_{x} \sin \theta}{R_{x}}\right)
$$

where $\frac{H V_{z}}{R_{x}}-\frac{R_{c} V_{x} \sin \theta}{R_{x}}$ is the Doppler frequency offset which is caused by the vertical velocity and squint angle. It should be corrected through the correction function as follows.

$$
H_{1}=\exp \left[-j 4 \pi \frac{f_{r}+f_{c}}{c}\left(-\frac{H V_{z}}{R_{x}}+\frac{R_{c} V_{x} \sin \theta}{R_{x}}\right) t_{a}\right]
$$

The result of Doppler frequency offset correction can be written as

$$
\begin{aligned}
& S_{1}\left(f_{r}, t_{a} ; R_{0}\right)=a_{r}\left(f_{r}\right) a_{a}\left(t_{a}\right) \exp \left(-j \frac{\pi f_{r}^{2}}{\gamma}\right) \\
& \exp \left[-j 4 \pi \frac{f_{r}+f_{c}}{c}\left(R_{x}+a_{1} t_{a}+k_{20} t_{a}^{2}+k_{30} t_{a}^{3}+k_{40} t_{a}^{4}\right)\right]
\end{aligned}
$$

Letting

$$
R_{1}\left(t_{a} ; R_{0}\right)=R_{c e n}+k_{2} t_{a}^{2}+k_{3} t_{a}^{3}+k_{4} t_{a}^{4}
$$

where, $R_{c e n}=2 R_{x}, k_{1}=2 a_{1}, k_{2}=2 k_{20}, k_{3}=2 k_{30}, k_{4}=2 k_{40}$.

After Doppler frequency offsetting, it can be expressed as

$$
\begin{aligned}
s_{1}\left(t_{r}, t_{a} ; R_{0}\right) & =a_{r}\left(t_{r}-\frac{R_{1}\left(t_{a} ; R_{0}\right)}{c}-\frac{k_{1} t_{a}}{c}\right) a_{a}\left(t_{a}\right) \\
& \exp \left[j \pi \gamma\left(t_{r}-\frac{R_{1}\left(t_{a} ; R_{0}\right)}{c}-\frac{k_{1} t_{a}}{c}\right)^{2}\right] \\
& \exp \left[-j \frac{2 \pi}{\lambda} R_{1}\left(t_{a} ; R_{0}\right)\right] \exp \left(-j 2 \pi \frac{f_{c} k_{1} t_{a}}{c}\right)
\end{aligned}
$$

Letting

$$
\begin{aligned}
& s_{A}\left(t_{r}, t_{a} ; R_{0}\right)=a_{r}\left(t_{r}-\frac{2 R_{1}\left(t_{a} ; R_{0}\right)}{c}\right) a_{a}\left(t_{a}\right) \\
& \exp \left[j \pi \gamma\left(t_{r}-\frac{2 R_{1}\left(t_{a} ; R_{0}\right)}{c}\right)^{2}\right] \exp \left[-j \frac{4 \pi}{\lambda} R_{1}\left(t_{a} ; R_{0}\right)\right]
\end{aligned}
$$

Equation (11) can be expressed as follows.

$$
s_{1}\left(t_{r}, t_{a} ; R_{0}\right)=s_{A}\left(t_{r}-\frac{k_{1} t_{a}}{c}, t_{a} ; R_{0}\right) \exp \left(-j 2 \pi \frac{f_{c} k_{1} t_{a}}{c}\right)
$$

Using the methods of POSP and Fresnel Integral, we get the range FT of (13)

$$
\begin{aligned}
S_{A}\left(f_{r}, t_{a} ; R_{0}\right)= & a_{r}\left(f_{r}\right) a_{a}\left(t_{a}\right) \exp \left(-j \frac{\pi f_{r}^{2}}{\gamma}\right) \\
& \exp \left[-j 2 \pi \frac{\left(f_{r}+f_{c}\right)}{c} R_{1}\left(t_{a} ; R_{0}\right)\right]
\end{aligned}
$$

According to the methods of POSP and series reversion [9], we get

$$
-\frac{c}{\left(f_{c}+f_{r}\right)} f_{a}=2 k_{2} t_{a}+3 k_{3} t_{a}^{2}+4 k_{4} t_{a}^{3}
$$

Using the method of series reversion [9] with (15), we obtain the stationary phase point

$$
t_{a}^{*}=A_{1}\left(-\frac{f_{a} c}{f_{c}+f_{r}}\right)+A_{2}\left(-\frac{f_{a} c}{f_{c}+f_{r}}\right)^{2}+A_{3}\left(-\frac{f_{a} c}{f_{c}+f_{r}}\right)^{3}
$$

where $A_{1}=\frac{1}{2 k_{2}}, A_{2}=-\frac{3 k_{3}}{8 k_{2}^{3}}, A_{3}=\frac{9 k_{3}^{2}-4 k_{2} k_{4}}{16 k_{2}^{5}}$.

Therefore, the azimuth FT of (14) is

$$
\begin{aligned}
S_{A}\left(f_{r}, f_{a} ; R_{0}\right)= & a_{r}\left(f_{r}\right) a_{a}\left(f_{a}\right) \exp \left(-j \frac{\pi f_{r}^{2}}{\gamma}\right) \\
& \exp \left\{-j 2 \pi\left[\frac{f_{c}+f_{r}}{c} R_{1}\left(t_{a}^{*} ; R_{0}\right)+f_{a} t_{a}^{*}\right]\right\}
\end{aligned}
$$

The phase of the second exponential can be expanded as 


$$
\begin{aligned}
& \frac{f_{c}+f_{r}}{c} R_{1}\left(t_{a}^{*} ; R_{0}\right)+f_{a} t_{a}^{*}=\frac{f_{c}+f_{r}}{c} R_{c e n} \\
& +\frac{A_{1}^{2} k_{2} c-A_{1} c}{f_{c}+f_{r}} f_{a}^{2} \\
& +\frac{-\left(-A_{2}+2 A_{1} A_{2} k_{2}+A_{1}^{3} k_{3}\right) c^{2}}{\left(f_{c}+f_{r}\right)^{2}} f_{a}^{3} \\
& +\frac{\left(-A_{3}+2 A_{1} A_{3} k_{2}+A_{2}^{2} k_{2}+A_{1}^{4} k_{4}+3 A_{1}^{2} A_{2} k_{3}\right) c^{3}}{\left(f_{c}+f_{r}\right)^{3}} f_{a}^{4} \\
& +\frac{-\left(2 A_{2} A_{3} k_{2}+3 A_{1}^{2} A_{3} k_{3}+3 A_{1} A_{2}^{2} k_{3}+4 A_{1}^{3} A_{2} k_{4}\right) c^{4}}{\left(f_{c}+f_{r}\right)^{4}} f_{a}^{5}+\cdots
\end{aligned}
$$

According to the properties of FT, the 2-D FT of $s_{1}\left(t_{r}, t_{a} ; R_{0}\right)$ is

$$
S_{1}\left(f_{r}, f_{a} ; R_{0}\right)=S_{A}\left(f_{r}, f_{a}+\left(f_{c}+f_{r}\right) \frac{k_{1}}{c} ; R_{0}\right)
$$

The effect of the high order terms of $f_{a}$ for phase is far less than $\pi / 4$, so we keep the terms of (18) up to the first 4th order of $f_{a}$. Then (19) can be written as

$$
S_{1}\left(f_{r}, f_{a} ; R_{0}\right)=a_{r}\left(f_{r}\right) a_{a}\left(f_{a}\right) \exp \left[j \varphi\left(f_{r}, f_{a}\right)\right]
$$

where, the phase function $\varphi\left(f_{r}, f_{a}\right)$ can be expanded as

$$
\begin{aligned}
\varphi\left(f_{r}, f_{a}\right)= & -2 \pi \frac{f_{c}+f_{r}}{c} R_{c e n}-\frac{\pi f_{r}^{2}}{\gamma} \\
& +2 \pi \frac{c}{4 k_{2}\left(f_{c}+f_{r}\right)}\left[f_{a}+\left(f_{c}+f_{r}\right) \frac{k_{1}}{c}\right]^{2} \\
& +2 \pi \frac{c^{2} k_{3}}{8 k_{2}^{3}\left(f_{c}+f_{r}\right)^{2}}\left[f_{a}+\left(f_{c}+f_{r}\right) \frac{k_{1}}{c}\right]^{3} \\
& +2 \pi \frac{c^{3}\left(9 k_{3}^{2}-4 k_{2} k_{4}\right)}{64 k_{2}^{5}\left(f_{c}+f_{r}\right)^{3}}\left[f_{a}+\left(f_{c}+f_{r}\right) \frac{k_{1}}{c}\right]^{4}
\end{aligned}
$$

And, the term $\frac{1}{f_{c}+f_{r}}$, and its expressions of square and cubic value can be also expanded as follows, respectively.

$$
\left\{\begin{array}{l}
\frac{1}{f_{c}+f_{r}} \approx \frac{1}{f_{c}}\left(1-\frac{f_{r}}{f_{c}}+\frac{f_{r}^{2}}{f_{c}^{2}}-\frac{f_{r}^{3}}{f_{c}^{3}}\right) \\
\frac{1}{\left(f_{c}+f_{r}\right)^{2}} \approx \frac{1}{f_{c}^{2}}\left(1-2 \frac{f_{r}}{f_{c}}+3 \frac{f_{r}^{2}}{f_{c}^{2}}-4 \frac{f_{r}^{3}}{f_{c}^{3}}\right) \\
\frac{1}{\left(f_{c}+f_{r}\right)^{3}} \approx \frac{1}{f_{c}^{3}}\left(1-3 \frac{f_{r}}{f_{c}}+6 \frac{f_{r}^{2}}{f_{c}^{2}}-10 \frac{f_{r}^{3}}{f_{c}^{3}}\right)
\end{array}\right.
$$

Then, (21) can be written as follows.

$$
\varphi\left(f_{r}, f_{a}\right)=\varphi_{0}\left(f_{a}\right)+\varphi_{1}\left(f_{a}\right) f_{r}+\varphi_{2}\left(f_{a}\right) f_{r}^{2}+\varphi_{3}\left(f_{a}\right) f_{r}^{3}
$$

Each of these phase terms are list as follows.

$$
\begin{aligned}
\varphi_{0}\left(f_{a}\right)= & -\frac{2 \pi f_{c} R_{c e n}}{c}+\frac{\pi f_{c} k_{1}^{2}}{2 c k_{2}}+\frac{\pi f_{c} k_{1}^{3} k_{3}}{4 c k_{2}^{3}}+\frac{\pi f_{c}\left(9 k_{3}^{2}-4 k_{2} k_{4}\right) k_{1}^{4}}{32 k_{2}^{5} c} \\
& +\frac{\pi k_{1} f_{a}}{k_{2}}+\frac{3 \pi k_{1}^{2} k_{3} f_{a}}{4 k_{2}^{3}}+\frac{\pi\left(9 k_{3}^{2}-4 k_{2} k_{4}\right) k_{1}^{3} f_{a}}{8 k_{2}^{5}} \\
& +\frac{\pi c f_{a}^{2}}{2 f_{c} k_{2}}+\frac{3 \pi c k_{1} k_{3} f_{a}^{2}}{4 k_{2}^{3} f_{c}}+\frac{\pi 3 c\left(9 k_{3}^{2}-4 k_{2} k_{4}\right) k_{1}^{2} f_{a}^{2}}{16 k_{2}^{5} f_{c}} \\
& +\frac{\pi c^{2} k_{3} f_{a}^{3}}{4 f_{c}^{2} k_{2}^{3}}+\frac{\pi c^{2}\left(9 k_{3}^{2}-4 k_{2} k_{4}\right) k_{1} f_{a}^{3}}{8 k_{2}^{5} f_{c}^{2}} \\
& +\frac{\pi c^{2}\left(9 k_{3}^{2}-4 k_{2} k_{4}\right) f_{a}^{4}}{32 k_{2}^{5} f_{c}^{3}}
\end{aligned}
$$

$$
\begin{aligned}
\varphi_{1}\left(f_{a}\right)= & -\frac{2 \pi R_{c e n}}{c}+\frac{\pi k_{1}^{2}}{2 c k_{2}}+\frac{\pi k_{1}^{3} k_{3}}{4 c k_{2}^{3}}+\frac{\pi\left(9 k_{3}^{2}-4 k_{2} k_{4}\right) k_{1}^{4}}{32 k_{2}^{5} c} \\
& -\frac{\pi c f_{a}^{2}}{2 k_{2} f_{c}^{2}}-\frac{3 \pi c k_{1} k_{3} f_{a}^{2}}{4 k_{2}^{3} f_{c}^{2}}-\frac{3 \pi c\left(9 k_{3}^{2}-4 k_{2} k_{4}\right) k_{1}^{2} f_{a}^{2}}{16 k_{2}^{5} f_{c}^{2}} \\
& -\frac{\pi c^{2} k_{3} f_{a}^{3}}{2 k_{2}^{3} f_{c}^{3}}-\frac{\pi c^{2}\left(9 k_{3}^{2}-4 k_{2} k_{4}\right) k_{1} f_{a}^{3}}{4 k_{2}^{5} f_{c}^{3}} \\
& -\frac{3 \pi c^{3}\left(9 k_{3}^{2}-4 k_{2} k_{4}\right) f_{a}^{4}}{32 k_{2}^{5} f_{c}^{4}} \\
\varphi_{2}\left(f_{a}\right)= & -\frac{\pi}{\gamma} \\
& +\frac{\pi c f_{a}^{2}}{2 k_{2} f_{c}^{3}}+\frac{3 \pi c k_{1} k_{3} f_{a}^{2}}{4 k_{2}^{3} f_{c}^{3}}+\frac{3 \pi c\left(9 k_{3}^{2}-4 k_{2} k_{4}\right) k_{1}^{2} f_{a}^{2}}{16 k_{2}^{5} f_{c}^{3}} \\
& +\frac{3 \pi c^{2} k_{3} f_{a}^{3}}{4 k_{2}^{3} f_{c}^{4}}+\frac{3 \pi c^{2}\left(9 k_{3}^{2}-4 k_{2} k_{4}\right) k_{1} f_{a}^{3}}{8 k_{2}^{5} f_{c}^{4}} \\
& +\frac{3 \pi c^{3}\left(9 k_{3}^{2}-4 k_{2} k_{4}\right) f_{a}^{4}}{16 k_{2}^{5} f_{c}^{5}} \\
\varphi_{3}\left(f_{a}\right)= & -\frac{\pi c f_{a}^{2}}{2 f_{c}^{4} k_{2}}-\frac{3 \pi c k_{3} k_{1} f_{a}^{2}}{4 f_{c}^{4} k_{2}^{3}}-\frac{3 \pi c\left(9 k_{3}^{2}-4 k_{2} k_{4}\right) k_{1}^{2} f_{a}^{2}}{16 f_{c}^{4} k_{2}^{5}} \\
& -\frac{\pi c^{2}\left(9 k_{3}^{2}-4 k_{2} k_{4}\right) k_{1} f_{a}^{3}}{2 f_{c}^{5} k_{2}^{5}}-\frac{\pi c^{2} k_{3} f_{a}^{3}}{f_{c}^{5} k_{2}^{3}} \\
& -\frac{5 \pi c^{3}\left(9 k_{3}^{2}-4 k_{2} k_{4}\right) f_{a}^{4}}{16 f_{c}^{6} k_{2}^{5}}
\end{aligned}
$$

In (23), $\varphi_{0}\left(f_{a}\right)$ is azimuth modulation term and has no relationship with $f_{r} \cdot \varphi_{1}\left(f_{a}\right)$ is the linear item coefficient of $f_{r}$. $\varphi_{2}\left(f_{a}\right)$ is the range chirp rate, and $\varphi_{3}\left(f_{a}\right)$ is the changing rate of the range chirp rate.

B . Imaging Algorithm

The flow chart of the algorithm is shown in Fig. 2.

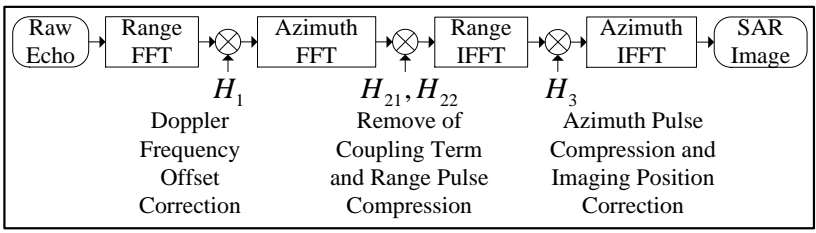

Fig. 2 The flow chart of the imaging algorithm

In (24), The linear item coefficient of $f_{a}$ is $-2 \pi\left(-\frac{k_{1}}{2 k_{2}}-\frac{3 k_{1}^{2} k_{3}}{8 k_{2}^{3}}-\frac{\left(9 k_{3}^{2}-4 k_{2} k_{4}\right) k_{1}^{3}}{16 k_{2}^{5}}\right.$, which indicates the azimuth position of the target. In order to getting the correct azimuth position, we need to make imaging position correction during azimuth compression. The term $-2 \pi R_{c e n} / c$ in (25) indicates the range position of the target, and the remaining terms in (25) are RCM which are also being corrected.

The steps of the diving and squint missile-borne SAR are as follows.

Step1. Getting $S\left(f_{r}, t_{a} ; R_{0}\right)$ through range FT of (5);

Step2. Correcting the Doppler frequency offset of $S\left(f_{r}, t_{a} ; R_{0}\right)$ by multiplying $H_{1}$; 
Step3. Getting 2-D spectrum $S_{1}\left(f_{r}, f_{a} ; R_{0}\right)$ through azimuth FT of $S_{1}\left(f_{r}, t_{a} ; R_{0}\right)$;

Step4. Removing the coupling term of $S_{1}\left(f_{r}, f_{a} ; R_{0}\right)$ by

$$
H_{21}=\exp \left[-j\left(\varphi_{1}\left(f_{a}\right)+R_{c e n} 2 \pi / c\right) f_{r}\right] \exp \left[-j \varphi_{3}\left(f_{a}\right) f_{r}^{3}\right]
$$

And getting the result of range pulse compression by

$$
H_{22}=\exp \left[-j \varphi_{2}\left(f_{a}\right) f_{r}^{2}\right]
$$

Then, we get $S_{1}\left(t_{r}, f_{a} ; R_{0}\right)$ through range IFT.

Step5. Getting the result of azimuth pulse compression by

$$
H_{3}=\exp \left[-j\left(\varphi_{0}\left(f_{a}\right)-\pi k_{1} f_{a} / k_{2}\right)\right]
$$

After azimuth IFT, we get the focusing SAR image.

When calculating $a_{1}, R_{c}, w_{1}, w_{2}, w_{3}$ and $w_{4}$, the values of $x_{0}$ and $y_{0}$ can be replaced by the coordinates $X_{c}$ and $Y_{c}$ of the imaging center.

\section{IV . Simulation Results}

We present some missile-borne SAR images generated by the proposed method in this paper. All the simulation results are shown on slant range plane. The designed scene consist some point targets, and the system parameters are list in TABLE 1.

TABLE 1 The simulation parameters

\begin{tabular}{|l|l|}
\hline Parameters & Numerical Value \\
\hline Carrier frequency $f_{c}$ & $35 \mathrm{GHz}$ \\
\hline Pulse duration $T_{r}$ & $5 \mu \mathrm{s}$ \\
\hline Bandwidth $B_{r}$ & $75 \mathrm{MHz}$ \\
\hline Imaging center coordinate $\left(X_{c}, Y_{c}, 0\right)$ & $(0,4000 \mathrm{~m}, 0)$ \\
\hline Initial flight altitude $H$ & $10000 \mathrm{~m}$ \\
\hline Initial Velocity $\left(V_{x}, V_{y}, V_{z}\right)$ & $(2000 \mathrm{~m} / \mathrm{s}, 0,-100 \mathrm{~m} / \mathrm{s})$ \\
\hline Acceleration $\left(a_{x}, a_{y}, a_{z}\right)$ & $\left(-50 \mathrm{~m} / \mathrm{s}^{2}, 0,-9.8 \mathrm{~m} / \mathrm{s}^{2}\right)$ \\
\hline Antenna Azimuth length $D$ & $1 \mathrm{~m}$ \\
\hline Sampling frequency $f_{s}$ & $200 \mathrm{MHz}$ \\
\hline PRF & $20 \mathrm{kHz}$ \\
\hline Squint angle & $42^{\circ}$ \\
\hline
\end{tabular}

Fig. 3 shows the results of pulse compression responses of point target. Fig. 3(a) shows the azimuth compression of point target, and Fig. 3(b) shows the azimuth compression of point target. Fig. 4 shows the contour plot of a point target. The quality parameters are list in TABLE 2 . The simulation results illustrate focusing performance of the method proposed in this paper meets the requirements of missile-borne SAR in diving and squint mode. The algorithm uses only four Fourier transforms and four complex multiplications, without any interpolation operation, and the operation efficiency is greatly improved.

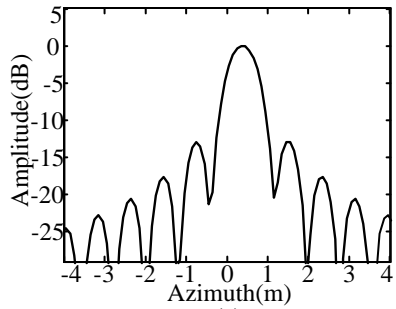

(a)

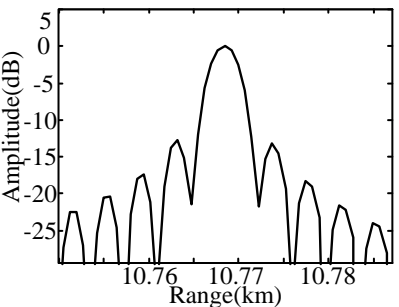

(b)
Fig. 3 The results of one point target. (a) Magnitude of Azimuth slices. (b) Magnitude of Range slices.

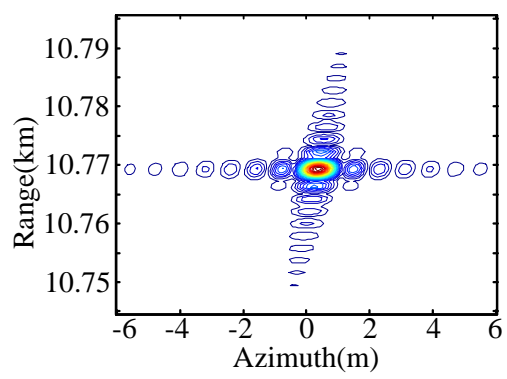

Fig. 4 Contour plot of the point target.

TABLE 2 Point target quality parameters

\begin{tabular}{|l|l|l|}
\hline Parameters & Range direction & Azimuth direction \\
\hline Resolution $(\mathrm{m})$ & 2 & 0.5 \\
\hline PSLR $(\mathrm{dB})$ & -13.19 & -12.93 \\
\hline ISLR(dB) & -10.01 & -9.94 \\
\hline
\end{tabular}

\section{Conclusions}

This paper presents an imaging algorithm for the diving and squint missile-borne SAR. The algorithm derives the 2-D frequency expression about echo signal of point target using the method of series reversion, and divides the RCM correction into main range walk correcting in time domain and remaining RCM correcting in range frequency-azimuth Doppler frequency domain. After the improvements about the current imaging algorithm of missile-borne squint SAR, we get a focused image. The point targets simulation results are presented to demonstrate its accuracy and validity of the proposed algorithm.

\section{References}

[1] Li Daojing, Zhang Linxi, Yu Bianzhang, "Research on active radar imaging seeker," Modern Radar, vol.5, pp. 12-15, 2003.

[2] Zhu Mingbo, Zhao Zhenbo, Li Xiangping, "Trajectory optimization for missile-borne SAR imaging phase via gauss pseudospectral method," IEEE CIE International Conference on Radar, pp. 867-870., 2011.

[3] Yi Yusheng, Zhang Linrang, Liu Nan, Liu Xin, Shen Dong, "An efficient imaging algorithm for missile-borne side-looking SAR," IET International Radar Conference, pp. 1-4, 2009.

[4] Zhou Song, Bao Min, Zhou Peng, Xing Mengdao, Bao Zheng, "An imaging algorithm for missile-borne SAR with downward movement based on azimuth nonlinear chirp scaling," Journal of Electronics \& Information Technology, vol. 33, No.6, pp. 1420-1426,2011.

[5] Zhang Donglei, Wang Feng, Li Yachao, "Improved chirp scaling algorithm for missile-borne SAR with high squint," Journal of Astronautics, vol. 32, No.6, pp. 1359-1364, 2011. 
[6] Bao Zheng, Xing Mengdao, Wang Tong, Radar Imaging Technolody, Bejing: Publishing House of Electronics Industry, 2005, pp.146-151.

[7] Huaying Xie, Hongzhong Zhao, Qiang Fu, "Taylor expansion and its application in missile-borne SAR imaging," APSAR, pp. 426-430, 2009.

[8] Ian G.Cumming, Frank H.Wong, Digital Processing of Synthetic Aperture Radar Data Algorithms and Implementation, Norwood, MA: Artech House, 2005.

[9] Yew Lam Neo, Frank H.Wong, Iam G.Cumming, "Processing of azimuth-invariant bistatic SAR data using the range Doppler algorithm," IEEE Trans on Geoscience and Remote Sensing, vol.46, No.1, pp. 14-21, 2008. 